\title{
Systems analysis of the familial cardiomyopathy signaling network
}

\author{
Ali Khalilimeybodi ${ }^{1}$, Jeffrey H. Omens ${ }^{2}$, Andrew D. McCulloch ${ }^{2}$, Jeffrey J. Saucerman ${ }^{1,3 *}$ \\ ${ }^{1}$ Department of Biomedical Engineering, University of Virginia, Charlottesville, Virginia, United States of America \\ ${ }^{2}$ Departments of Bioengineering and Medicine, University of California, San Diego, La Jolla, California, United States of America \\ ${ }^{3}$ Robert M. Berne Cardiovascular Research Center, University of Virginia, Charlottesville, Virginia, United States of America \\ * Corresponding author
}

\begin{abstract}
Familial cardiomyopathy is a precursor of heart failure and sudden cardiac death. Over the past several decades, researchers have discovered numerous gene mutations primarily in sarcomeric and cytoskeletal proteins causing two different disease phenotypes: hypertrophic (HCM) and dilated (DCM) cardiomyopathies. However, molecular mechanisms linking genotype to phenotype remain unclear. Here, we employ a systems approach by integrating experimental findings into a cohesive signaling network to scrutinize genotype to phenotype mechanisms. We developed a predictive model of the HCM/DCM signaling network utilizing a logic-based differential equations approach and evaluated model performance in predicting experimental data from four contexts (HCM, DCM, pressure overload, and volume overload). The model has an overall prediction accuracy of $83.8 \%$ with higher accuracy in the HCM context (90\%) compared with DCM (75\%). Global sensitivity analysis identified key reactions of the signaling network, with calcium activation of myofilament force development and calcium-calmodulin kinase signaling ranking the highest. We performed a structural revision analysis to identify missing interactions in HCM/DCM signaling and found some potential reactions controlling calcium regulatory proteins. Combination pharmacotherapy analysis predicted that downregulation of signaling components such as calcium, titin and its associated proteins, growth factor receptors, and PI3K-AKT could inhibit myocyte growth in HCM. In DCM, PI3K-AKT-NFAT downregulation combined with upregulation of Ras/ERK12 or titin or Gq protein could attenuate cardiac remodeling. The influence of existing medications on cardiac growth in HCM and DCM contexts was simulated by the model. This HCM/DCM signaling model demonstrates utility in investigating genotype to phenotype mechanisms in familial cardiomyopathy.
\end{abstract}

\section{Introduction}

Familial cardiomyopathy, a common cause of heart failure (HF) and sudden cardiac death ${ }^{1,2}$, is a class of genetic disorder typically caused by mutations primarily in myocytes ${ }^{3}$. Dilated (DCM) and hypertrophic (HCM) cardiomyopathies are two main clinical phenotypes of familial cardiomyopathy, each affecting roughly from 1:500 to 1:250 individuals globally ${ }^{4-6}$. To date, apart from transplantation, there are no effective cures for patients suffering from these cardiac muscle diseases ${ }^{7}$. Morphologically, DCM is characterized by dilation of the left ventricular chamber with systolic dysfunction. In contrast, HCM is associated with asymmetric cardiac growth, thickened ventricular walls, and diastolic dysfunction.

Even though numerous gene mutations that underlie HCM and DCM have been discovered ${ }^{8}$, our understanding of mechanisms leading from genotype to phenotype remains incomplete. This lack of 
understanding may originate from a significant diversity in mutations causing the same phenotype, especially in $\mathrm{DCM}^{6}$, or in the dependency of the cardiac phenotype on both environmental factors and genetic mutations $s^{9,10}$. However, the field has lacked systems approaches that integrate the relevant molecular signaling findings into a cohesive framework to scrutinize genotype to phenotype mechanisms. As cardiac signaling plays a key role in the development of DCM and $\mathrm{HCM}^{11}$, understanding HCM/DCM signaling can elucidate major molecular players contributing to intrinsic variability and complexity of genotype-phenotype relations in familial cardiomyopathy. It may also help to identify new drug targets and design more efficacious long-term pharmacological treatments for familial cardiomyopathy.

Although mutations in more than 100 genes have been linked to familial cardiomyopathy, sarcomeric gene mutations are the most common cause ${ }^{12}$. These mutations usually alter the dynamics of intracellular calcium, myofilament force generation, or both. In many cases, change in myofilament $\mathrm{Ca}^{2+}$-sensitivity is a specific alteration leading to contractile dysregulation. Often, DCM-associated mutations decrease myofilament sensitivity to calcium and lead to faster calcium dissociation from sarcomeres during relaxation and lower active force generation. In contrast, most $\mathrm{HCM}$-linked mutations increase $\mathrm{Ca}^{2+}$ sensitivity following by extended force relaxation time and higher contractile force ${ }^{11,13}$. Altered calcium handling and active force subsequently affect associated cardiac signaling pathways resulting in cardiac growth and remodeling.

In a significant study by Davis et al. ${ }^{11}$, the authors utilized a series of mouse models, in vitro and in vivo, combined with a computational model of myocyte contraction to study familial cardiomyopathy. They revealed that integrated active force magnitude over time is a major predictor of cardiac phenotype during hypertrophic and dilated cardiomyopathies. They also showed that calcineurin $(\mathrm{CaN}) /$ nuclear factor of activated $\mathrm{T}$ cells (NFAT) and Ras/extracellular signal-regulated kinase 1/2 (ERK12) pathways are involved in developing HCM and DCM phenotypes by altering cardiomyocytes' mass and eccentricity. Other signaling pathways such as phosphoinositide-3-kinase(PI3K)/protein kinase B (AKT) ${ }^{14}$, and nitric oxide $(\mathrm{NO}) /$ protein kinase $\mathrm{G}(\mathrm{PKG})^{15}$ have been also reported in the development of familial cardiomyopathy phenotypes. Moreover, titin and its associated proteins and regulators such as FHL1, FHL2, and RBM20 act like a mechanosensor in cardiomyocytes and play a significant role in the initiation and development of HCM and DCM through regulation of both molecular pathways and cardiac passive stiffness. While experimental models are necessary to identify mechanisms linking the genotype to phenotype in familial cardiomyopathy, challenges such as complex cardiac signaling pathways ${ }^{16}$, modified biomechanical stresses ${ }^{14}$, and neurohormonal system activity ${ }^{17}$, make them insufficient to fully determine the underlying mechanisms in HCM and DCM.

Computational models have been used to improve our understanding of mechanisms involved in multiple scales of cardiomyopathy ${ }^{18}$. However, at the cellular and tissue levels, while numerous signaling and finite element models have been developed for cardiac growth and remodeling ${ }^{19}$, to the authors' knowledge, none of them investigated familial cardiomyopathies ${ }^{18}$. Hence, in this study, we constructed and validated a computational model of familial cardiomyopathy signaling. The model links the mutation-induced variations of calcium sensitivity to alteration of myocytes' mass and eccentricity in hypertrophic and 
dilated cardiomyopathies. The model predicts the major reactions regulating the cardiomyopathy phenotype and provides new insights on potential drug targets to decrease adverse cardiac growth and remodeling in familial cardiomyopathy.

\section{Results}

\section{1- A conceptual model of cardiac remodeling in response to $\mathrm{HCM} / \mathrm{DCM}$ mutations and mechanical loading}

In terms of cell morphology, the main difference between HCM and DCM is cardiomyocyte eccentricity. HCM mutations cause cardiomyocyte growth through the addition of sarcomeres in parallel that increases myocyte cross-sectional area without significant changes in length. On the contrary, DCM mutations lead to an increase in the myocyte length by adding sarcomeres in series. Hence, HCM and DCM mutations decrease and increase myocyte eccentricity, respectively. Among the primary factors of familial cardiomyopathy regulating myocyte eccentricity, several studies demonstrated the integrated active force as a key factor ${ }^{11,20}$. HCM and DCM affect cardiomyocyte active force through changing myocyte contractility $^{21}$. The negative correlation between myocardial active force and eccentricity is not limited to familial cardiomyopathy. In the pressure overload condition, elevated afterload leads to increased active force $^{22}$, and decreases myocardial eccentricity ${ }^{23}$ (Fig. 1a).

In the context of volume-overload, increased cardiac passive force regulates myocyte eccentricity. After volume overload, there is a shift of the end-diastolic volume to the right in the ventricular pressure-volume loop, which results in higher cardiac tissue elasticity and passive force. This increased passive force then initiates a cardiac response leading to cardiac dilation ${ }^{23}$. Interestingly, titin stiffness is a major contributor to cardiomyocyte passive stiffness and force and is positively correlated with active force in HCM, DCM, and pressure-overload conditions. Yet, titin stiffness is negatively correlated with eccentricity except in some reported cases in volume-overload hypertrophy (Fig. 1a). These observations suggest a potential mediating role for titin stiffness in genotype to phenotype mechanisms. By considering the above factors and their interrelations, we propose a conceptual model (Fig. 1a) that can explain the distinct cardiac responses observed in various contexts. The model emphasizes the role of titin and biomechanical factors such as active and passive forces in regulating familial cardiomyopathies. However, in cardiomyocytes, a complex signaling network conveys the effects of gene mutations to changes in cell eccentricity and stiffness and is responsible for interactions illustrated in the conceptual model.

\section{2- Predictive computational model of HCM/DCM signaling network}

To reconstruct the familial cardiomyopathy signaling network (Fig. 1b), we first identified the major signaling modules contributing to cardiomyocyte responses in familial cardiomyopathy from the literature. Davis et al. ${ }^{11}$ indicated the myofilament force-generation module, CaN-NFAT, and ERK12 pathways regulate mass and eccentricity in familial cardiomyopathy. Other studies showed signaling pathways such as PI3K-AKT ${ }^{14,21}$, NO-PKG ${ }^{15,24}$, calcium ${ }^{25}, \beta$-adrenergic ${ }^{13,26}$, and downstream pathways of Gq protein ${ }^{27}$ and growth factor receptors ${ }^{28,29}$ could play a role in familial cardiomyopathy. Moreover, titin has been described as a regulator of familial cardiomyopathy via its effects on cardiomyocyte stiffness, force generation ${ }^{30}$, and mechanotransduction ${ }^{31}$. Then, we reconstructed the molecular 
interactions between identified signaling components by employing the cardiac signaling networks developed by Ryall et al. ${ }^{32}$ and Tan et al., ${ }^{33}$ which investigated cardiac hypertrophy and mechanosignaling, respectively. Due to limited data availability, we included components with demonstrated roles in cardiac growth and remodeling and available experimental data or perturbations in studied contexts (Fig. 1b).

a
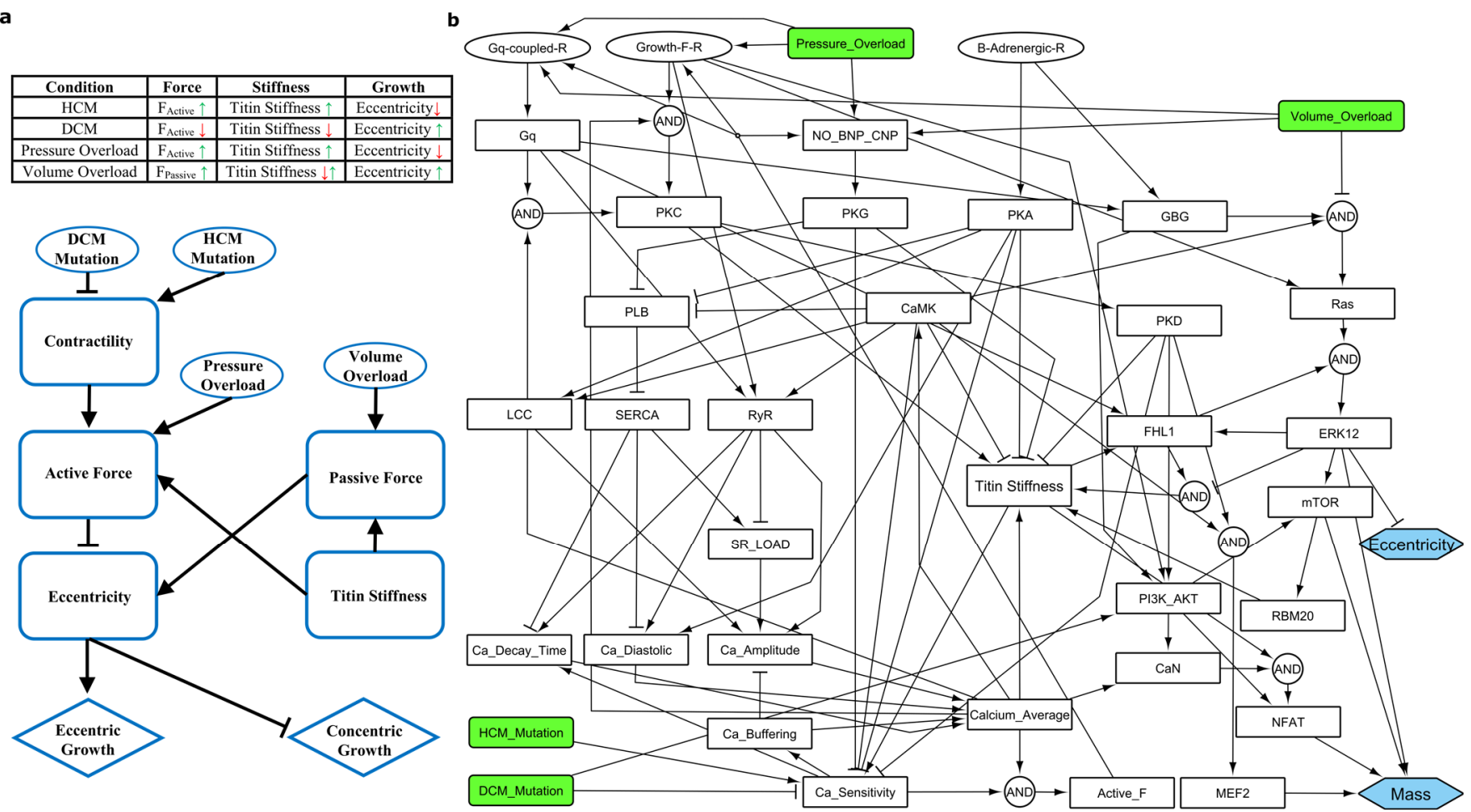

Fig. 1 Reconstruction of the familial cardiomyopathy signaling network. (a) Context-dependent responses of cardiomyocytes in cardiac growth and remodeling is simplified by a conceptual model of HCM/DCM. Interactions between cardiomyocyte active force, passive force, titin stiffness, and eccentricity regulate cardiac phenotype. (b) HCM/DCM signaling network. Four model inputs (green) are linked to two model outputs in the myocyte (blue) through 33 signaling species (white) and 82 reactions.

Since titin and calcium modules were less-developed in previous models ${ }^{32,33}$, we reconstructed them with more cell signaling detail. As shown in previous studies ${ }^{34}$ and proposed by the conceptual model (Fig. 1a), titin could play a major role in regulating cardiac signaling in familial cardiomyopathy. Several kinases like $\mathrm{Ca}^{2+} /$ calmodulin-dependent protein kinase (CaMK), protein kinase D (PKD), protein kinase A (PKA), ERK12 phosphorylate titin at different positions and decrease titin stiffness. In contrast, phosphorylation by protein kinase $\mathrm{C}$ (PKC) increases titin stiffness ${ }^{24}$. RNA binding motif protein 20 (RBM20) regulates alternative splicing of titin, which changes the ratio of compliant N2BA to stiff N2B isoform and subsequently affects titin stiffness. Titin also can affect ERK12 and CaN-NFAT pathways through its associated proteins including four and a half LIM domain (FHL) proteins, FHL1 and FHL2 ${ }^{35}$. The intracellular calcium cycling module is a significant part of active force generation in cardiomyocytes, and its diverse response has been reported in familial cardiomyopathy ${ }^{11,25}$. Also, mutations in calcium regulating proteins such as phospholamban (PLB) might lead to familial cardiomyopathy ${ }^{36}$. Therefore, we 
added a simplified calcium module comprising its related characteristics such as sarcoplasmic reticulum (SR) $\mathrm{Ca}^{2+}$ load, $\mathrm{Ca}^{2+}$ amplitude, buffering, diastolic level, and decay time, as well as its regulating proteins and channels such as PLB, SR $\mathrm{Ca}^{2+}$-ATPase (SERCA), ryanodine receptors (RyR), and L-type calcium channel (LTCC). It is noteworthy that we added pressure overload and volume overload stimuli to our model to explore the dynamics of familial cardiomyopathy signaling influenced by biomechanical signals. This enables the model to simulate the influence of some medications on cardiac phenotype capturing both their molecular and hemodynamic effects.

Overall, the HCM/DCM signaling network model includes 39 nodes (four inputs: HCM mutation, DCM mutation, pressure overload, volume overload; two outputs: mass and eccentricity; and 33 signaling species) connected by 82 reactions (S1 Table). Applying a logic-based differential equations (LDE) approach $^{37}$, we converted the HCM/DCM signaling interaction graph (Fig. 1b) into a predictive computational model. The normalized activity of each node is computed utilizing ordinary differential equations with saturating Hill functions. We used continuous logical "AND" and "OR" gates to combine the influence of multiple reactions on a signaling node. Similar to our previously published models of

cardiac signaling ${ }^{16,32}$, constant default values have been used for all network parameters. We used a smaller default value for reaction weight of inhibitory reactions reducing calcium sensitivity and titin stiffness, to prevent saturation in unstimulated conditions. Based on the availability of quantitative experimental data, individual parameters of the model can be calibrated subsequently to provide more quantitative predictions. By employing Netflux software, we automatically generated a system of LDEs and simulated HCM/DCM signaling dynamics in MATLAB.

\section{3- Model performance in predicting context-specific qualitative data}

To evaluate the model performance in predicting cardiac morphology and variations of signaling nodes in four contexts (hypertrophic and dilated cardiomyopathies, pressure-overload, and volume-overload hypertrophy), we curated context-specific experimental data from the literature. All data were categorized as a decrease, no change, and increase according to their statistical significance compared to the control in each study (S2 Table). As diversity in test data is crucial for assessing the performance of complex models, we employed the following strategies in obtaining data from the literature. First, we tried to obtain data, as much as possible, for all key signaling nodes of the model in studied contexts such as HCM and DCM. Second, various experimental data with and without intermediate perturbations such as inhibition, overexpression, and multi-stimuli were obtained. We also included overexpression data (no additional context) and Gq context data in the validation dataset due to their importance. Third, we included all reported responses for signaling nodes in a context, such as including both calcium amplitude increase and decrease in DCM (Fig. 2).

Simulated activity changes of network nodes after HCM mutation, DCM mutation, pressure overload, and volume overload with/without perturbation of various nodes are compared with associated validation data in Fig. 2. The HCM/DCM model with default parameters has a total prediction accuracy of $83.8 \%$ $(134 / 160)$ across all contexts. The model has its highest prediction accuracy in the pressure overload 
context with $92.3 \%(24 / 26)$, followed by HCM with $90.2 \%(37 / 41)$, DCM with 75\% (36/48), and the volume overload context with $66.6 \%$ (16/24) validation accuracy.

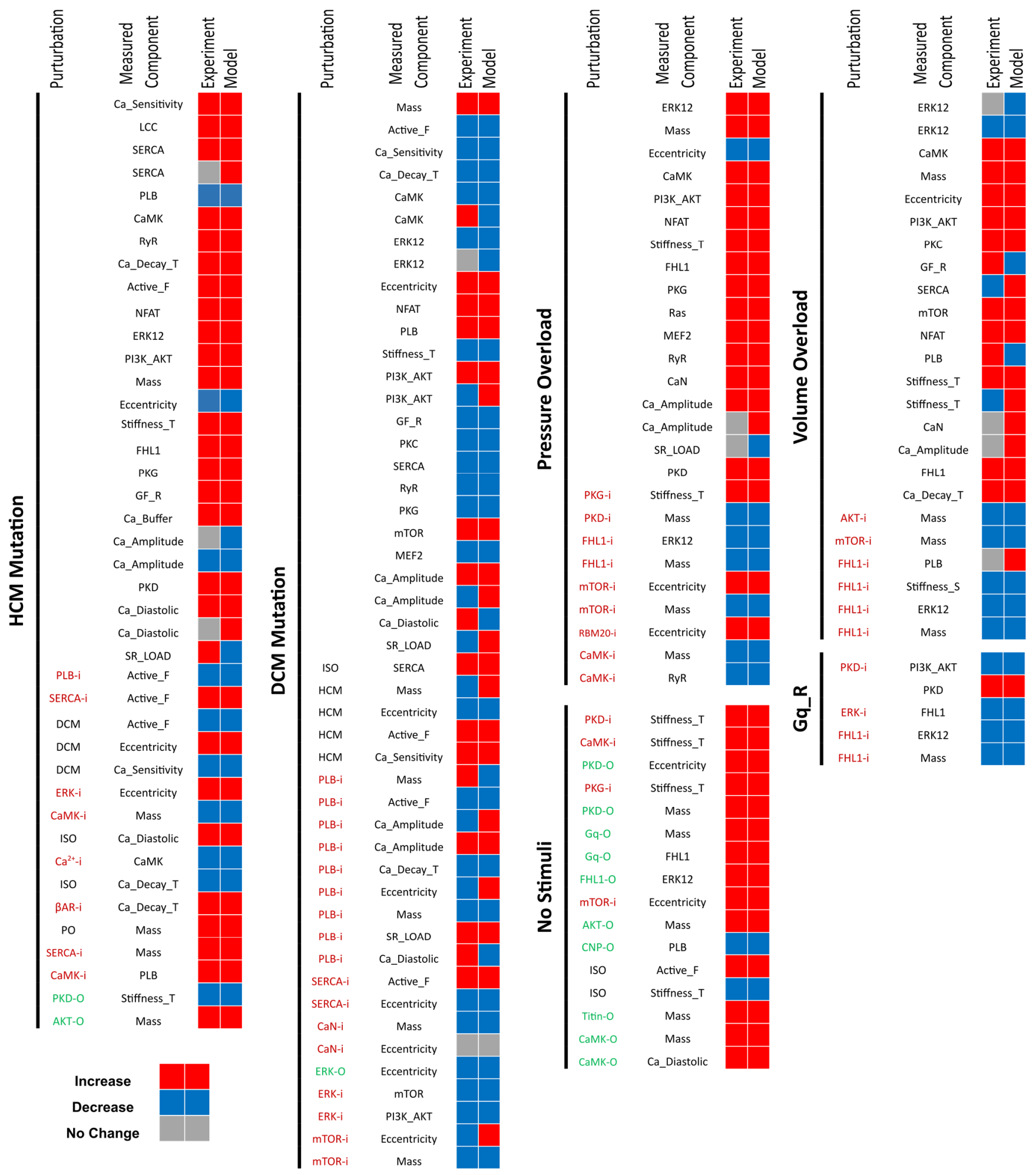

Fig. 2 Classified qualitative validation illustrates HCM/DCM model performance in different contexts. Comparison of model predictions with 160 experimental data points from literature (S2 table). Inhibitory and overexpression perturbations are 
illustrated by $-\mathrm{i}$ (red) and $-\mathrm{o}$ (green), respectively. Relative changes greater than the in-silico threshold $(0.001 \%)$ and statistically significant in comparison with the control have been considered for the model and experimental results, respectively.

It is noteworthy that in some cases discordant responses were reported for the same experiment, which leads to a decrease in the model prediction accuracy regardless of model actual performance. These nodes are mostly located in the calcium module. For example, while Davis et al. ${ }^{11}$ reported an increase in $\mathrm{Ca}^{2+}$ transient amplitude in DCM, Robinson et al. ${ }^{21}$ and Liu et al. ${ }^{38}$ reported the opposite, which can be a result of different gene mutations ${ }^{6}$, or altered mechanisms regulating calcium level ${ }^{39}$. In the case of discordant experimental data, all data were included. The DCM context has the highest number of discordant experimental data. In addition to $\mathrm{Ca}^{2+}$ transient amplitude, CaMK, ERK12, and PI3K-AKT responses to DCM mutations are also contradicting in the literature. However, changes in mass and eccentricity as the main indicators of the cardiac phenotype are consistent in each context and correctly predicted by the model.

As predicted by the model and supported by experimental observations (Fig. 2), HCM mutations increase calcium sensitivity, integrated active force, CaMK, PKG, PI3K-AKT, ERK12, NFAT, and titin stiffness (S2 Table). HCM mutation consequently leads to an increase in mass and a decrease in eccentricity. In DCM mutation, the model largely predicted opposite changes in CaMK, PKG, ERK12, and titin stiffness, but there are still increases in PI3K-AKT, NFAT, and mass. The model predicted attenuated changes in eccentricity after concurrent HCM and DCM input signals. As reported by Powers et al. ${ }^{20}$ and Davis et al. ${ }^{11}$, double-transgenic murine models containing both HCM and DCM gene mutations have more normal cardiac morphology compared to DCM or HCM murine models. Regarding titin stiffness, the model predicts an increase in titin stiffness after HCM mutation, pressure overload, and volume overload consistent with experimental observations reported by Herwig et al. ${ }^{27}$, Michel et al. ${ }^{40}$, and Mohammad et al. ${ }^{41}$, respectively. In contrast, as predicted by the model, DCM mutations lead to a decrease in titin stiffness $^{42}$.

\section{4- Identification of key network regulators and potential interactions in cardiac remodeling}

A key step in finding effective drugs for a disease is to determine how alteration in individual reactions will affect the cell response. Determination of reactions with the highest impact reveals the significance of each pathway in regulating the cell response and minimizes the efforts and costs of follow-up experiments by avoiding non-significant pathways. Although local sensitivity analysis can provide useful information ${ }^{43}$, it does not capture nonlinear interactions between reactions in a complex network like HCM/DCM signaling. Thus, we performed Morris global sensitivity analysis to identify reactions with the highest impact on model prediction accuracy. By selecting the reactions' weight $(\mathrm{W})$ as the variable parameter, we calculated the Morris index $\left(\mu^{*}\right)$ and standard deviation for each reaction in the model (Fig. 3a). Reactions with higher scores $\left(\mu^{*}>2\right)$ were visualized on the HCM/DCM signaling network (Fig. 3b). As illustrated in Fig. 3, 24 of 82 reactions of the model significantly affect the model prediction accuracy.

Although non-significant reactions may partly contribute to signal transduction in cardiomyopathy, modifying them has a negligible effect on the overall response of the HCM/DCM signaling network. For 
example, CaMK, PKA, PKG, ERK12, and PKC all phosphorylate titin and modify its stiffness. However, based on the results, only PKA and PKG effects on titin stiffness have significant contributions to $\mathrm{HCM} / \mathrm{DCM}$ signaling. It is noteworthy that significance in the Morris sensitivity analysis is a relative concept. Based on the sensitivity analysis from Fig. 3, reactions modeling the effects of calcium sensitivity and calcium average on the active force have the highest impact on HCM/DCM signaling network. This is consistent with experimental studies by Powers et al. ${ }^{20}$ and Davis et al. ${ }^{11}$ that reported significant changes in cardiac phenotype after a modification in calcium sensitivity and active force in HCM or DCM contexts. Moreover, as predicted by the model and supported by experimental observations, activation of CaMK by calcium ${ }^{25}$, titin contribution to active force through calcium sensitivity ${ }^{30}$, activation of PKA by $\beta$-adrenergic receptors ${ }^{44}$, PKG by nitric oxide ${ }^{45}$, and ERK12 by Ras and FHL1 ${ }^{46}$ could significantly affect cardiac response in cardiomyopathy.

a

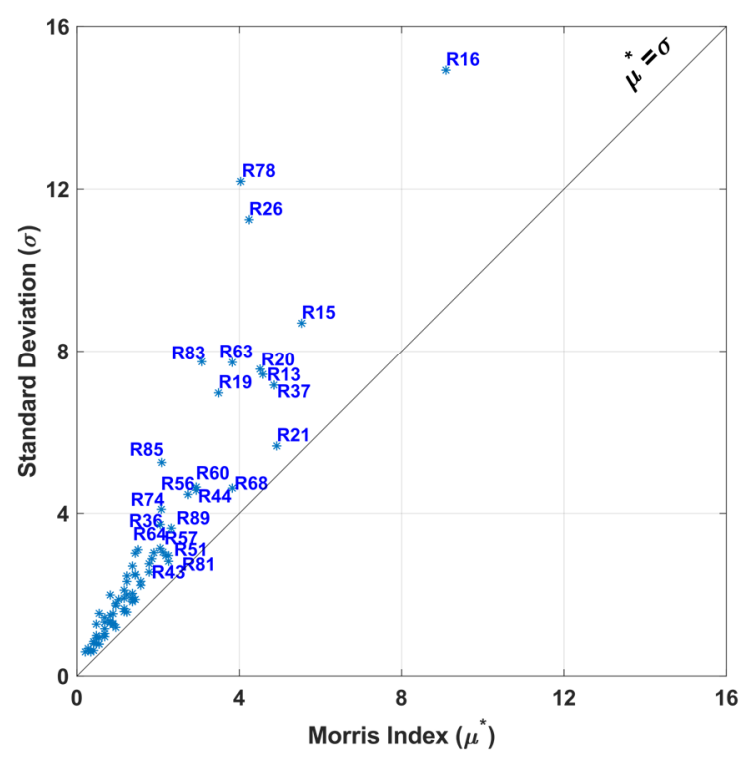

b

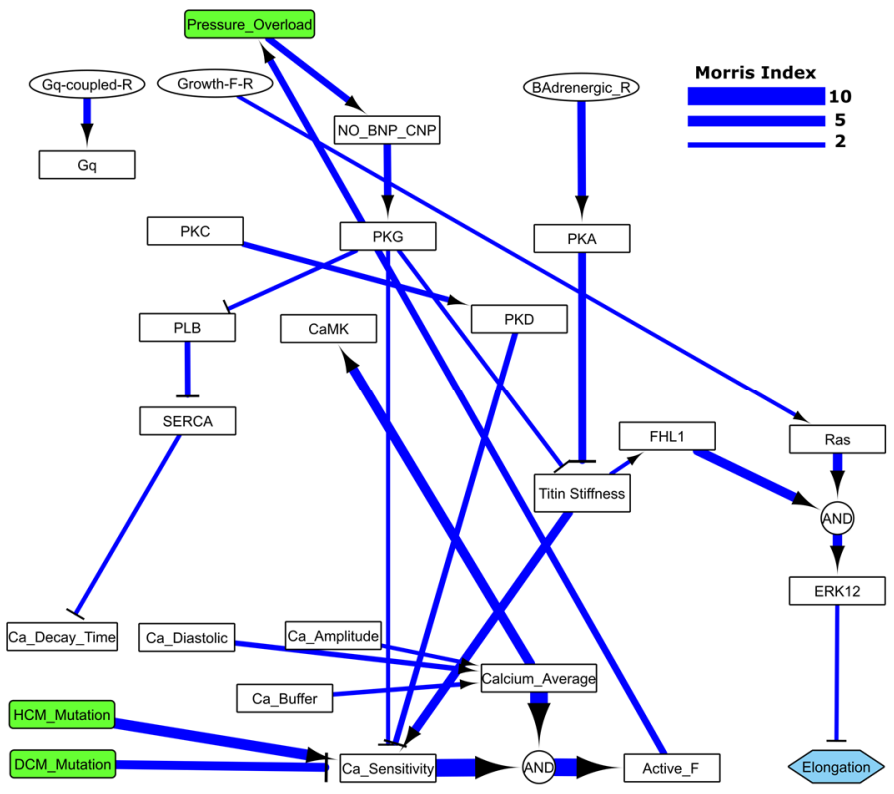

Fig. 3 Morris global sensitivity analysis identifies key signaling reactions in familial cardiomyopathy. (a) Morris index $\left(\mu^{*}\right)$ depicts important $\left(\mu^{*}>2\right)$ and less-important reactions in HCM/DCM signaling model. Greater Morris index means more influence on model validation percent. A larger $\sigma$ to $\mu^{*}$ ratio demonstrates a more nonlinear effect. (b) Visualization of important reactions in familial cardiomyopathy signaling based on their ranks from Morris sensitivity analysis. The thicker arrows illustrate the larger Morris index.

Even though the HCM/DCM signaling model has a reasonably good validation accuracy (83.8\%), there are some incorrect predictions. Apart from discordant responses for the same experiment, imprecise model parameters and structure could influence model prediction accuracy. Because of limited quantitative data availability, we used default parameters for the model, which inevitably reduces the model prediction accuracy. Concerning model structure, we developed the model by employing identified mediators of cardiac signaling in familial cardiomyopathy with their known interactions in cardiac signaling. Thus, new yet-to-be-discovered interactions may exist in cardiomyopathy signaling. To address this, we applied a structural revision method, CLASSED, we developed previously ${ }^{16}$ to find new potential interactions that improve model validation accuracy. We screened all possible interactions between the model components 
by a systematic, one-by-one adding of new reactions between signaling nodes through the "OR" gate and from each node to the existing reactions by an "AND" gate.

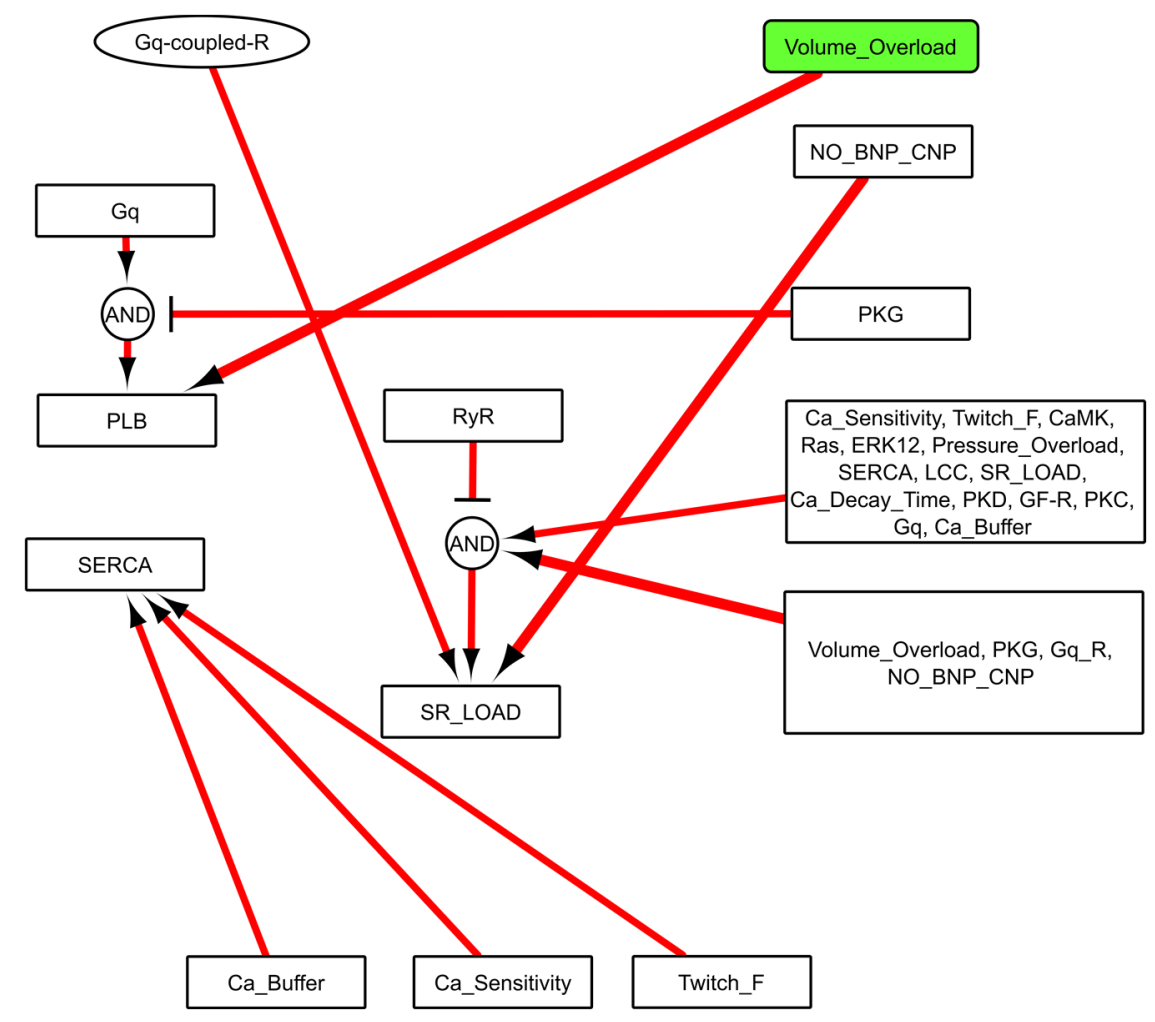

Fig. 4 Structural revision analysis suggests new potential interactions in HCM/DCM signaling. The thicker arrows illustrate a larger improvement in model prediction accuracy.

Figure 4 displays the inferred reactions that improve the model prediction accuracy by more than one percent. These inferred reactions indicate potential causal relations between signaling components and provide a baseline for further experiments. According to Fig. 4, Gq protein and volume overload stimulus may affect calcium signaling by raising PLB activity. As shown in HCM/DCM model (Fig. 1b), PKC is a common downstream component of both $\mathrm{Gq}$ protein and volume overload signals and may be the mediator of these inferred reactions. Braz et al. ${ }^{47}$ showed that PKC affects PLB phosphorylation and activity. PLB phosphorylation increased in PKC $\alpha$-deficient mice, whereas it decreased in transgenic mice overexpressing PKC $\alpha$.

The results also suggest contributions of several signaling nodes such as NO and PKG in $\mathrm{SR} \mathrm{Ca}^{2+}$ load regulation (Fig. 4). In the model, NO leads to phosphorylation of PLB by PKG and increases $\mathrm{Ca}^{2+}$ uptake by SERCA, which consequently raises $\mathrm{SR} \mathrm{Ca}^{2+}$ load and $\mathrm{Ca}^{2+}$ transient amplitude. Thus, the inferred reactions point to a more pronounced positive effect of $\mathrm{NO}-\mathrm{PKG}$ on $\mathrm{SR} \mathrm{Ca}^{2+}$ load in familial cardiomyopathy through other parallel pathways and/or higher reaction weights (W) for the existing pathway. This is in agreement with experimental studies. While both positive and negative effects of NOPKG on SR $\mathrm{Ca}^{2+}$ load and $\mathrm{Ca}^{2+}$ transient through mediators like SERCA, RyR, sodium/hydrogen exchanger (NHE), PKA, PLB have been reported ${ }^{48-50}$, its positive effect seems to be dominant in 
cardiomyopathy signaling ${ }^{51}$. Previous studies reported increased activity of PKG and SERCA with elevated $\mathrm{SR} \mathrm{Ca}^{2+}$ load in $\mathrm{HCM}$, and the opposite response in $\mathrm{DCM}^{24,26,52}$.

\section{5- Evaluation of new and existing pharmacotherapies}

A growing number of clinical trials on combination therapies ${ }^{53}$, and their higher performance in treating cardiac disease like heart failure ${ }^{54}$, make them an important consideration for drug development. Thus, we analyzed the efficiency of all possible drug targets in the context of HCM and DCM mutations through combinatory inhibition and overexpression of model signaling nodes by changing the Ymax parameter from 1.0 to 0.5 and 1.5 , respectively.

a

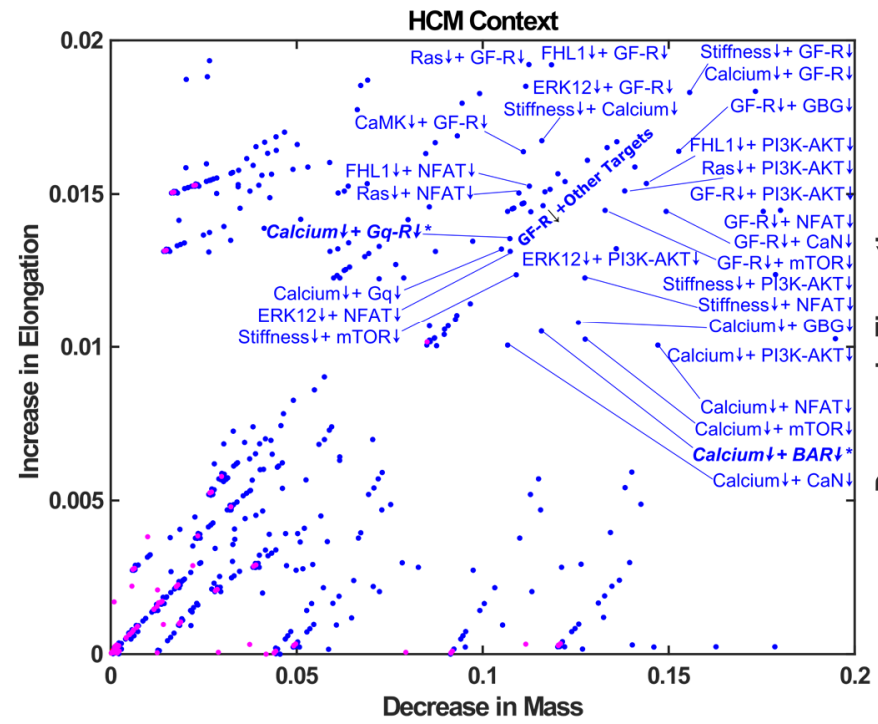

b

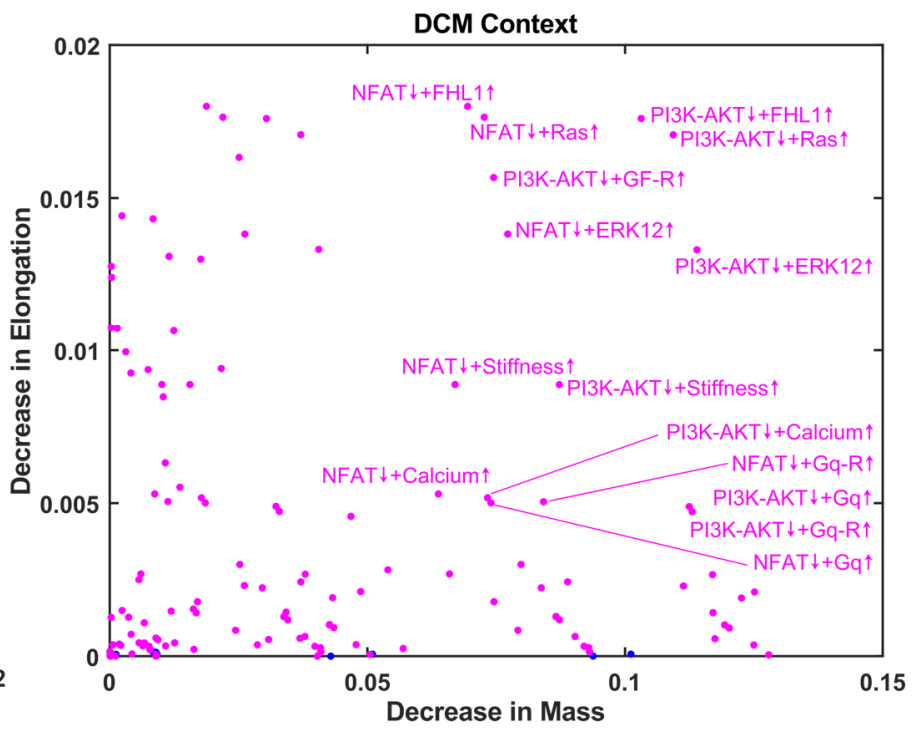

Fig. 5 Efficacy of combination pharmacotherapies on familial cardiomyopathy. All pairwise combinations of reducing $(Y \max =0.5)$ or increasing $(Y \max =1.5)$ nodes' activity and their subsequent effects on myocardial mass and eccentricity in the context of HCM mutations (a) and DCM mutations (b). (*) shows two combinatory drug targets (Calcium+ Gq-R, Calcium $+\beta A R$ ) that are among conventional treatment strategies.

As shown in Fig. 5a, drugs that knockdown two targets have the highest effect in decreasing mass and increasing eccentricity in the HCM context. In DCM, combined pharmacotherapies including concurrent overexpression and knockdown of two separate nodes provide the best results in preventing cardiac growth and remodeling in DCM. For example, in the HCM context, paired knockdown of growth factor receptors (GF-R) with calcium, titin stiffness, or G beta-gamma complex (GBG) leads to the highest decrease in mass and increase in eccentricity. In general, combined knockdown of nodes such as GF-R, PI3K-AKT, NFAT, ERK12, mechanistic target of rapamycin (mTOR), titin stiffness, and calcium are predicted as potential treatment strategies for the HCM context.

In the DCM context (Fig. 5b), the number of potential drug targets is less than the HCM context, and usually, they are limited to knockdown of NFAT or PI3K-AKT with overexpression of Gq protein associated receptors (Gq-R), calcium, ERK12, FHL1, and titin stiffness. While there are no experimental studies that investigated combination pharmacotherapies for familial DCM or HCM, some studies showed the effectiveness of targeting one of the predicted signaling nodes by the model. As shown in Fig. 5, the 
model predicts that ERK12 overexpression or inhibition can contribute to preventing mutation-induced changes in cell eccentricity in DCM and HCM, respectively. Likewise, Davis et al. ${ }^{11}$ observed that mitogen-activated protein kinase kinase (MEK) overexpression (upstream of ERK12) in rat cardiomyocytes decreases the DCM-induced increase in cell eccentricity. They also showed that MEK inhibition reverses the decrease in cell eccentricity in rat cardiomyocytes with HCM mutation. In support of model predictions (Fig. 5b), Dhandapany et al. ${ }^{55}$ showed that inhibition of the AKT pathway rescued the DCM phenotype. Furthermore, the model predicted two of the known targets of existing heart failure drugs. As shown in Fig. 5a, combined knockdown of calcium with $\beta$ AR or calcium with Gq-R could prevent the HCM phenotype. Ho et al. ${ }^{56}$ and Semsarian et al. ${ }^{57}$ showed in their studies that diltiazem, a $\mathrm{Ca}^{2+}$ channel blocker, could prevent the familial hypertrophic cardiomyopathy in human patients and mouse model, respectively.

Numerous drugs have been developed to relieve the symptoms of and prevent heart failure progression ${ }^{58}$. While some partly prevented cardiac growth and remodeling in heart failure ${ }^{59}$, a few studies focused on the effects of current drugs on cardiac remodeling in familial cardiomyopathies. Hence, we employed the HCM/DCM signaling model to predict the outcomes of available medications on cardiac growth and remodeling through changes in cardiomyocyte mass and eccentricity (Table 1).

Table 1 Efficacy of existing pharmacotherapies on familial cardiomyopathy

\begin{tabular}{|c|c|c|c|c|c|c|c|}
\hline Drug & $\begin{array}{c}\text { Hemodynamic } \\
\text { Effects }\end{array}$ & Context & $\begin{array}{l}\text { Simulated } \\
\text { Conditions }\end{array}$ & $\begin{array}{l}\text { Mass } \\
\text { Model }\end{array}$ & $\begin{array}{c}\text { Eccentricity } \\
\text { Model }\end{array}$ & $\begin{array}{l}\text { Mass } \\
\text { Exp. }\end{array}$ & $\begin{array}{l}\text { Eccentricity } \\
\text { Exp. }\end{array}$ \\
\hline \multirow{2}{*}{$\begin{array}{l}\boldsymbol{\beta} \text {-blockers } \\
\text { (carvedilol, } \\
\text { metoprolol, } \\
\text { Propranolol) }\end{array}$} & \multirow{2}{*}{$\begin{array}{c}\text { Afterload } \downarrow \\
\text { Preload - }\end{array}$} & $\mathrm{HCM}$ & \multirow{2}{*}{$\begin{array}{l}\beta A R: 1 \rightarrow 0.5 \\
P^{*}: 0.5 \rightarrow 0\end{array}$} & $\downarrow$ & $\uparrow$ & - & - \\
\hline & & DCM & & $\downarrow$ & $\uparrow$ & - & - \\
\hline \multirow{2}{*}{$\begin{array}{c}\text { Ca2+ channel } \\
\text { blockers (verapamil, } \\
\text { diltiazem, } \\
\text { candesartan) }\end{array}$} & \multirow{2}{*}{$\begin{array}{l}\text { Afterload } \downarrow \\
\text { Preload - }\end{array}$} & $\mathrm{HCM}$ & \multirow{2}{*}{$\begin{array}{l}\text { LCC: } 1 \rightarrow 0.5 \\
\text { PO: } 0.5 \rightarrow 0\end{array}$} & $\downarrow$ & $\uparrow$ & $\downarrow^{56}$ & $\uparrow^{56}$ \\
\hline & & DCM & & $\downarrow$ & $\uparrow$ & - & - \\
\hline \multirow{2}{*}{$\begin{array}{c}\text { Cardiac glycosides } \\
\text { (digoxin) }\end{array}$} & \multirow{2}{*}{$\begin{array}{l}\text { Afterload - } \\
\text { Preload - }\end{array}$} & $\mathrm{HCM}$ & \multirow{2}{*}{$\begin{array}{l}\mathrm{Ca}^{2+} \text { Amplitude: } \\
1 \rightarrow 1.5 \\
\text { RBM20: } 1 \rightarrow 0.5\end{array}$} & $\uparrow$ & $\downarrow$ & - & - \\
\hline & & DCM & & $\uparrow$ & $\downarrow$ & - & - \\
\hline \multirow{2}{*}{$\begin{array}{c}\text { Angll blockers: } \\
\text { ARBs and ACEs } \\
\text { (valsartan, Lisinopril, } \\
\text { losartan) }\end{array}$} & \multirow{2}{*}{$\begin{array}{l}\text { Afterload } \downarrow \\
\text { Preload } \downarrow\end{array}$} & $\mathrm{HCM}$ & \multirow{2}{*}{$\begin{array}{c}\text { Gq-R: } 1 \rightarrow 0.5 \\
\text { PO: } 0.5 \rightarrow 0 \\
\text { VO*: } 0.5 \rightarrow 0\end{array}$} & $\downarrow$ & $\uparrow$ & $\downarrow^{60,61}$ & NS $^{61,62}$ \\
\hline & & DCM & & $\downarrow$ & $\uparrow$ & - & - \\
\hline \multirow{2}{*}{$\begin{array}{c}\text { Nitric oxide } \\
\text { activators (sacubitril, } \\
\text { vericiguat) }\end{array}$} & \multirow{2}{*}{$\begin{array}{l}\text { Afterload } \downarrow \\
\text { Preload } \downarrow\end{array}$} & $\mathrm{HCM}$ & \multirow{2}{*}{$\begin{array}{l}\text { NO: } 1 \rightarrow 1.5 \\
\text { PO: } 0.5 \rightarrow 0 \\
\text { VO: } 0.5 \rightarrow 0\end{array}$} & $\downarrow$ & $\uparrow$ & - & - \\
\hline & & DCM & & $\downarrow$ & $\uparrow$ & - & - \\
\hline \multirow{2}{*}{$\begin{array}{l}\text { Myosin inhibitors } \\
\text { (mavacamten, MYK- } \\
224 \text { ) }\end{array}$} & \multirow{2}{*}{$\begin{array}{l}\text { Afterload - } \\
\text { Preload - }\end{array}$} & $\mathrm{HCM}$ & \multirow{2}{*}{$\begin{array}{l}\text { Active_F: } \\
1 \rightarrow 0.5\end{array}$} & $\downarrow$ & $\uparrow$ & $\downarrow^{63}$ & $\uparrow^{63}$ \\
\hline & & DCM & & $\downarrow$ & $\uparrow$ & - & - \\
\hline \multirow{2}{*}{$\begin{array}{l}\text { Myosin and non- } \\
\text { myosin Activators } \\
(\mathrm{MYK}-491, \mathrm{ACT}-1)\end{array}$} & \multirow{2}{*}{$\begin{array}{l}\text { Afterload - } \\
\text { Preload - }\end{array}$} & $\mathrm{HCM}$ & \multirow{2}{*}{$\begin{array}{c}\text { Active_F: } \\
1 \rightarrow 1.5\end{array}$} & $\uparrow$ & $\downarrow$ & - & - \\
\hline & & $\mathrm{DCM}$ & & $\uparrow$ & $\downarrow$ & - & - \\
\hline
\end{tabular}

* PO: Pressure Overload, VO: Volume Overload 
We categorized drugs based on their mechanism of action and included hemodynamic effects to increase simulation accuracy. As shown in Table 1, the model predicts a decrease in mass and an increase in eccentricity in HCM and DCM contexts for most medications. Even though a reduced mass is a positive outcome for patients with HCM and DCM, increased eccentricity would be detrimental for DCM patients. In contrast, cardiac glycosides such as digoxin and myosin activators such as MYK-491 could lead to an elevated mass and decreased eccentricity. In agreement with model predictions, clinical studies on patients with hypertrophic cardiomyopathy indicated $\mathrm{Ca}^{2+}$ channel blockers like diltiazem ${ }^{56}$ and myosin inhibitors like mavacamten ${ }^{63}$ could decrease cardiac mass and increase cardiomyocyte eccentricity based on measured chamber dimensions. The angiotensin receptor blockers such as losartan ${ }^{60}$ and candesartan ${ }^{61}$ also decreased cardiac mass, but their effect on cardiomyocyte eccentricity was non-significant.

\section{Discussion}

Here, we proposed a conceptual model to explain distinct phenotypes in familial cardiomyopathy. Inspired by previous experimental studies, we illustrated in the conceptual model how cardiac active and passive forces and titin stiffness are linked to myocyte eccentricity in different conditions. By considering the central role of titin and its stiffness in regulating cardiac response in familial cardiomyopathy, we developed and validated a predictive model of the HCM/DCM signaling network to understand the underlying mechanisms of genotype to phenotype in familial cardiomyopathy. The model sensitivity analysis revealed influential reactions regulating HCM/DCM signaling network and suggested some new potential interactions that increase model prediction accuracy. We performed a combination pharmacotherapy analysis through in-silico knockdown and overexpression of signaling nodes and discovered some potential drug targets to inhibit cardiac growth and morphology changes in familial HCM and DCM. We also simulated the effects of current medications on cardiomyocyte eccentricity and mass and evaluated their effectiveness. This HCM/DCM signaling model provides a framework to investigate genotype to phenotype mechanisms in familial cardiomyopathy. The model yields insights into new potential drugs to decrease the maladaptive cardiac growth and remodeling in familial cardiomyopathy as a main precursor of heart failure.

\section{1- Titin and cardiomyopathy}

In addition to the active force module, the model analysis indicated titin could play a significant role in regulating HCM/DCM phenotypes. Titin is considered an important mediator in the initiation and development of familial cardiomyopathy ${ }^{64}$. However, underlying mechanisms by which titin links genotype to phenotype in familial cardiomyopathy are not fully understood. As shown in the model (Fig. 1b), kinases such as PKD, PKG, CaMK, PKA, and ERK12 phosphorylate titin and reduce its stiffness, which is contrary to PKC that increases titin stiffness by phosphorylation ${ }^{65}$. In HCM, while the model predicted an increase in the activity of PKD, PKG, CaMK, and ERK12 kinases consistent with experimental data ${ }^{15,25,27}$, both model and experimental studies showed no decrease in titin stiffness ${ }^{27,66}$. This highlights the importance of other factors such as elevated PKC or alteration in titin splicing as causes of the long-term increase in titin stiffness during HCM. In DCM, the model predicted a decrease in the activity of all kinases except PKA. However, experimental studies depicted discordant kinases activity in 
ERK12 $2^{11,21}$ and CaMK activity in DCM $^{21,37}$. These could be the results of diversity in DCM gene mutations $^{6}$, transient response of ERK12 activity, and activation of CaMK by other pathways ${ }^{67}$. The projected decrease in titin stiffness by model and patients with dilated cardiomyopathy ${ }^{42}$ suggests the significance of PKC-dependent phosphorylation of titin's PEVK domain ${ }^{65}$ and other non-kinase regulators in altering titin stiffness in DCM.

RBM20 is a key player in controlling the splicing process of titin mRNA ${ }^{68}$, and RBM20 mutation results in DCM. Inhibiting RBM20 function leads to an increase in the more compliant N2BA titin isoform and decreases titin stiffness ${ }^{69}$. Rbm20 $\Delta$ RRM heterozygous mice have higher LV chamber compliance and enhanced exercise performance with normal systolic function. ${ }^{69}$ As shown in the HCM/DCM signaling model (Fig. 1b), mTOR could link PI3K-AKT and ERK12 pathways to RBM20. In all contexts, the model predicts an increase in mTOR activity, which is supported by an Ikeda et al. study of volume overload ${ }^{70}$ and a study by Yano et al. on DCM $^{71}$. Elevated mTOR activity could increase titin stiffness through RBM20 as observed in all contexts except DCM ${ }^{42}$. In DCM, decreased titin stiffness could be due to the elevated expression of compliant N2BA isoform ${ }^{42,72}$, which suggests the role of other regulators of the titin splicing process or stiffness in DCM patients. As shown in the model, calcium can modify titin stiffness, too. Recent studies displayed elevated titin force and stiffness in calcium-activated myofibrils ${ }^{73}$. While calcium alone raises titin stiffness ${ }^{74}$, its influence is small in comparison with an increase in titin stiffness after sarcomere activation ${ }^{75}$. Nishikawa et al. ${ }^{73}$ described that titin-thin filament interaction in active sarcomeres is mainly responsible for this calcium-dependent increase in titin stiffness. Thus, part of the change in titin stiffness, especially in DCM, could be due to altered calcium predicted by the model.

Furthermore, titin is a signal transducer in cardiac mechanotransduction ${ }^{76}$. Among the titin-associated proteins, FHL1 and FHL2 are decisive in HCM/DCM signaling and contribute to activation of ERK12 and NFAT pathways. In agreement with experimental data (Fig. 2), the model predicted an increase in FHL1 activity in HCM, pressure overload, and volume overload contexts, which then could increase ERK12 activity and regulate cardiomyocyte eccentricity. Regarding FHL2, HCM mutation results in decreased FHL2 mRNA and protein levels ${ }^{77}$ and thus attenuates its inhibitory effect on NFAT through binding to $\mathrm{CaN}^{78}$. Moreover, the model considers titin's contribution to active force development. Modifying the Frank-Starling mechanism by alteration of interfilament lattice spacing ${ }^{79}$, titin stiffening in muscle activation, and unfolding and refolding of titin IgG domains ${ }^{80}$ are potential mechanisms that could link titin to HCM and DCM phenotypes.

\section{2- CaMK: Integrator of calcium signaling in familial cardiomyopathy}

The multifunctional signaling molecule CaMK has been acknowledged as a master regulator of arrhythmias and maladaptive remodeling in cardiac diseases ${ }^{81}$. In cardiomyopathy, as predicted by the model (Fig. 3), CaMK acts as an integrator of altered calcium signals and regulates genotype to phenotype mechanisms. CaMK completes a control loop in calcium signaling by linking the changes in calcium transient to the activation of calcium cycling regulators such as SERCA, LCC, and RyR. Moreover, it alters cardiomyocyte stiffness and calcium sensitivity through titin and troponin phosphorylation, respectively, and can affect cardiomyocyte morphology through ERK12 and MEF2 ${ }^{82}$. Recently, various 
studies have presented CaMKII $\delta$ as a target of RBM20 that might be involved in RBM20 mutationinduced dilated cardiomyopathy ${ }^{83}$. While some studies reported the beneficial effects of CaMK modification ${ }^{84}$, altering CaMK activity as an effective treatment strategy in cardiomyopathy (Fig. 5) or even heart failure ${ }^{85}$ is challenging due to a large number of CaMK target proteins. Though a consistent increase in CaMK activity has been reported for the HCM context ${ }^{25,86}$, reported CaMK activity in the DCM context is variant ${ }^{21,38}$. This inconsistency may arise from diverse gene mutations leading to DCM that initiate different compensatory mechanisms to regulate calcium cycling and the level of disease progression. Robinson et al. ${ }^{21}$ studied functional consequences of three variant mutations in thin-filament regulatory proteins that led to DCM with reduced systolic $\mathrm{Ca}^{2+}$, SERCA, and CaMK activity. However, Liu et al. ${ }^{38}$ reported decreased SERCA and $\mathrm{Ca}^{2+}$ transients with increased CaMK activity after R25C-PLN mutation in the DCM.

Studies investigating DCM in patients often do not differentiate between various etiologies. Furthermore, data from patients with end-stage heart failure makes it harder to find the primary reason for the reported increase in CaMK activity ${ }^{87}$. To sum up, CaMK could be a key drug target in familial cardiomyopathy due to its central role in HCM/DCM signaling (Fig. 3). However, a wide range of mechanisms affecting CaMK activity in human dilated cardiomyopathy and heart failure ${ }^{82}$, and contradicting impacts of CaMK on different mediators of HCM/DCM signaling demands more specific studies on CaMK to accurately predict its therapeutic effects.

\section{3- Challenges in drug development}

The main challenges in developing drugs for familial cardiomyopathy are the significant diversity in the genotype to phenotype relationship and its unknown mechanisms. In this study, we explored part of these mechanisms concerning the cardiac signaling network and predicted some potential drug targets to decrease cardiac growth and remodeling in familial cardiomyopathy. According to the model predictions, inhibition of PI3K-AKT and/or NFAT seem the most effective targets for inhibition of elevated cardiac mass during HCM and DCM. In this regard, Fan et al. ${ }^{88}$ showed that DM-celecoxib as well as celecoxib, which inhibit AKT and then NFAT, improved left ventricular systolic functions and prevented cardiac remodeling and reduced mortality in DCM. Moreover, the efficacy of inhibiting PI3K-AKT and GF-Rs has been established in blunting hypertrophic response to exercise training ${ }^{89}$, pressure overload ${ }^{90}$, and neurohormonal factors ${ }^{91,92}$. The role of PI3K-AKT and EGFR inhibition in preventing cardiac fibrosis, a significant characteristic of HCM, has also been reported. The main concern in inhibiting GF-Rs and especially PI3K-AKT is their vital role in other cardiac functions such as cardiomyocyte proliferation, survival, and apoptosis ${ }^{93}$. mTOR is another potential drug target for HCM (Fig. 5). Rapamycin, a specific inhibitor of mTOR, attenuates or reverses cardiac growth and remodeling in pressure overload ${ }^{94}$ and $\mathrm{HCM}^{95}$ contexts. Altering titin stiffness is another effective treatment strategy in both HCM and DCM, predicted by the model (Fig. 5). While HCM mutations led to an elevated stiffness in both titin and cardiac tissue (ECM), the model results suggest that more compliant titin could partly reduce hypertrophic remodeling and subsequently improves its diastolic dysfunction. Methawasin et al. ${ }^{96}$ showed a significant improvement in diastolic dysfunction and exercise tolerance after reducing titin stiffness in pressureoverload hypertrophy leading to HFpEF. In DCM, model analysis suggests that an increase in titin 
stiffness could be beneficial by reducing cell eccentricity. Hutchinson et al. ${ }^{97}$ showed that an increase in myocardial stiffness in the volume overload context is beneficial and limits its eccentric remodeling.

\section{4- Limitations and future directions}

Although the model effectively predicts the variations of signaling pathways that lead to familial HCM and DCM, it has some limitations. The cardiac metabolic network plays a significant role in mediating genotype to phenotype in both familial cardiomyopathies, especially in their progression to heart failure ${ }^{98}$. In DCM, truncating mutations in the TTN gene was associated with a reduced rate of $\mathrm{O}_{2}$ consumption, significant ROS production, and transcriptional upregulation of mitochondrial oxidative phosphorylation system $^{99,100}$. Galata et al. ${ }^{101}$ reported an association between altered ERK12 signaling after LMNA gene mutation and changes in shape, fragmentation, and distribution of cardiomyocyte mitochondria. In HCM, deficient creatine kinase-phosphocreatine coupling in the hypercontractile myocardium led to diastolic dysfunction through inadequate ATP regeneration ${ }^{102}$. Moreover, fibrosis and structural variations in ECM during familial cardiomyopathy modify the biomechanical forces sensed by cardiomyocytes and affect phenotype development ${ }^{103}$. The above factors as well as mutations altering non-structural proteins influence the model performance in predicting possible scenarios. For the future, integrating the HCM/DCM signaling model with a cardiac metabolic network as well as incorporating variations in the cell mechanics and mechanotransduction in the model would be beneficial. Finally, we developed and validated the HCM/DCM signaling model, which provides new insights into the genotype-to-phenotype mechanisms in familial cardiomyopathy.

\section{Methods}

\section{1- Model construction}

A knowledge-based computational model of the HCM/DCM signaling network was developed from the literature. Main cardiac signaling modules were identified from over 40 peer-reviewed papers addressing the role of certain signaling modules in dilated or hypertrophic cardiomyopathy by in-vitro and invivo studies. Individual reactions between signaling nodes were then added, based on cardiac signaling networks developed by Ryall et al. ${ }^{32}$ and Tan et al. ${ }^{33}$ that modeled cardiac hypertrophy and mechanosignaling networks, respectively. In the calcium module, we focused on steady-state variations in the activity of its major regulators such as RyR, SERCA, PLB, LCC and their effects on calcium transient characteristics such as $\mathrm{Ca}^{2+}$ amplitude, diastolic level, decay time, and $\mathrm{Ca}^{2+}$ buffering and SR Load. Calcium average and active force nodes are integrated parameters to consider both the time and amplitude of their variations ${ }^{11}$.

We employed the logic-based differential equation (LDE) approach ${ }^{37}$ to build a predictive framework to explore HCM/DCM signaling dynamics. Activation of each node by its upstream reactions was modeled by a normalized Hill function. We modeled pathways crosstalk by continuous gates representing "OR" and "AND" logic 37 . The OR gates were used for reactions that modify the node regardless of others and the AND gates for reactions affecting each other. The reaction parameters in the model are reaction weight $(\mathrm{W}=0.5)$, half-maximal effective concentration $(\mathrm{EC} 50=0.5)$, and Hill coefficient $(\mathrm{n}=1.4)$. The time 
constant $(\tau=1)$, initial activation (Yinit $=0$ ) and maximal activation (Ymax $=1$ ) regulate the dynamics of signaling nodes. In the case of multiple inhibitory inputs for a node, such as titin stiffness or calcium sensitivity, the default reaction weight leads to an oversaturated node activity. Furthermore, a default weight for the reaction directly linking DCM-Mutation to PI3K-AKT activation makes PI3K-AKT insensitive to its other upstream regulators. Thus, we used a reaction weight of 0.1 for these reactions (S1 Table). The Netflux software (available at https://github.com/saucermanlab/ Netflux) has been used to generate a system of LDEs. Simulation of experimental conditions has been performed by the model validation module (available at https://github.com/mkm1712/Automated_Validation) in MATLAB software.

\section{2- Context-specific data collection}

Experimental data of familial hypertrophic and dilated cardiomyopathies, pressure overload, and volume overload conditions were manually curated from the literature. We acquired qualitative data at steady state or an appropriate time point from over 70 research articles with similar cell lines, and experimental conditions. Overall, 160 qualitative experimental data sets with and without overexpression and inhibition perturbations were obtained including 41 and 48 data points for HCM and DCM contexts, respectively. These data were either at steady state or suitable time points for nodes with transient activity. The contextspecific data, simulated conditions, and references are displayed in S2 Table.

\section{3- Global sensitivity analysis}

We employed a Morris global sensitivity analysis approach to identify signaling reactions with a greater influence on the model validity in familial cardiomyopathy. The Morris elementary effects method is a statistical method to screen models with many parameters or high computational cost for simulation. We utilized the Sampling for Uniformity (SU) strategy with input factor level equals to eight, oversampling size of 300, and trajectories' number equal to 16 to generate the sampling data using the EE sensitivity package developed by Khare et al. ${ }^{104}$. The reaction weight $(\mathrm{W})$ was selected as the variable parameter to compute the sensitivity measure, $\mu^{*}$, for each reaction. $\mu^{*}$ is the average of the elementary effects' absolute value to certify robustness against non-monotonic models but cannot capture nonlinear effects. Therefore, to account for interactions between factors or nonlinearities in the model, we computed $\sigma$ as the standard deviation of the elementary effects.

\section{Disclosures}

A.D.M. and J.H.O. are co-founders of and have equity interests in Insilicomed Inc. and serve as scientific advisors. A.D.M is also a co-founder of and scientific advisor to Vektor Medical, Inc. Some of their research grants have been identified for conflict of interest management based on the overall scope of the project and its potential benefit to these companies. These authors are required to disclose this relationship in publications acknowledging the grant support; however, the research subject and findings reported in this study did not involve the companies in any way and have no relationship with the business activities or scientific interests of either company. The terms of this arrangement have been reviewed and approved by the University of California San Diego in accordance with its conflict of interest policies. 


\section{References}

1. A, K. Molecular genetics and pathogenesis of cardiomyopathy. J. Hum. Genet. 61, 41-50 (2016).

2. MA, B. et al. Molecular profiling of dilated cardiomyopathy that progresses to heart failure. JCI insight $\mathbf{1}$, (2016).

3. EM, M., JR, G. \& MJ, P. Genetic mutations and mechanisms in dilated cardiomyopathy. J. Clin. Invest. 123, 19-26 (2013).

4. M, S., MR, T. \& L, M. Diagnosis, prevalence, and screening of familial dilated cardiomyopathy. Expert Opin. orphan drugs 3, 869-876 (2015).

5. A, B. et al. Complex roads from genotype to phenotype in dilated cardiomyopathy: scientific update from the Working Group of Myocardial Function of the European Society of Cardiology. Cardiovasc. Res. 114, 1287-1303 (2018).

6. RE, H., DJ, H. \& A, M. Dilated cardiomyopathy: the complexity of a diverse genetic architecture. Nat. Rev. Cardiol. 10, 531-547 (2013).

7. JC, T. et al. Targets for therapy in sarcomeric cardiomyopathies. Cardiovasc. Res. 105, 457-470 (2015).

8. M, S.-M., I, P.-S., JP, H. D. R. \& JR, G. Genetics of hypertrophic cardiomyopathy: A review of current state. Clin. Genet. 93, 3-14 (2018).

9. H, M., J, S. \& CE, S. Genetic causes of human heart failure. J. Clin. Invest. 115, 518-526 (2005).

10. T, E. \& L, C. Cardiomyopathy phenotypes in human-induced pluripotent stem cell-derived cardiomyocytes-a systematic review. Pflugers Arch. 471, 755-768 (2019).

11. J, D. et al. A Tension-Based Model Distinguishes Hypertrophic versus Dilated Cardiomyopathy. Cell 165, 1147-1159 (2016).

12. EM, M., DY, B. \& MJ, P. The genetic landscape of cardiomyopathy and its role in heart failure. Cell Metab. 21, 174-182 (2015).

13. H, W. et al. Modelling diastolic dysfunction in induced pluripotent stem cell-derived cardiomyocytes from hypertrophic cardiomyopathy patients. Eur. Heart J. 40, 3685-3695 (2019).

14. R, C. et al. A Contraction Stress Model of Hypertrophic Cardiomyopathy due to Sarcomere Mutations. Stem cell reports 12, 71-83 (2019).

15. LR, H. \& A, K.-K. Proteomic Analysis of Myocardia Containing the Obscurin R4344Q Mutation Linked to Hypertrophic Cardiomyopathy. Front. Physiol. 11, (2020).

16. A, K., AM, P., SLM, C. \& JJ, S. Context-specific network modeling identifies new crosstalk in $\beta$ adrenergic cardiac hypertrophy. PLoS Comput. Biol. 16, (2020).

17. F, T. et al. The influence of the angiotensin I converting enzyme genotype in familial hypertrophic cardiomyopathy varies with the disease gene mutation. J. Mol. Cell. Cardiol. 29, 831-838 (1997).

18. Y, A., JD, P., KJ, M. \& AD, M. Multiscale Models of Cardiac Muscle Biophysics and Tissue Remodeling in Hypertrophic Cardiomyopathies. Curr. Opin. Biomed. Eng. 11, 35-44 (2019).

19. K, Y. \& JW, H. Computational models of cardiac hypertrophy. Prog. Biophys. Mol. Biol. 159, 75-85 (2021).

20. JD, P. et al. Modulating the tension-time integral of the cardiac twitch prevents dilated cardiomyopathy in murine hearts. JCI insight 5, (2020).

21. P, R. et al. Dilated cardiomyopathy mutations in thin-filament regulatory proteins reduce contractility, suppress systolic $\mathrm{Ca} 2+$, and activate NFAT and Akt signaling. Am. J. Physiol. Heart Circ. Physiol. 319, H306-H319 (2020).

22. RJ, K., DF, T., P, S., F, van B. \& J, B. Interaction between afterload and contractility in the newborn heart: evidence of homeometric autoregulation in the intact circulation. J. Am. Coll. Cardiol. 25, 14281435 (1995).

23. FG, P. \& CM, T. Heart Plasticity in Response to Pressure- and Volume-Overload: A Review of Findings in Compensated and Decompensated Phenotypes. Front. Physiol. 11, (2020).

24. N, H., M, H. \& WA, L. Tampering with springs: phosphorylation of titin affecting the mechanical function of cardiomyocytes. Biophys. Rev. 9, 225-237 (2017).

25. P, R. et al. Hypertrophic cardiomyopathy mutations increase myofilament $\mathrm{Ca} 2+$ buffering, alter 
intracellular Ca 2+ handling, and stimulate Ca 2+-dependent signaling. J. Biol. Chem. 293, 10487-10499 (2018).

26. M, W., S, W. \& A, E.-A. Linking superinhibitory PLN mutations to CaMKII activation: a new arrhythmogenic mechanism in genetic DCM? Cardiovasc. Res. 107, 5-6 (2015).

27. M, H. et al. Modulation of Titin-Based Stiffness in Hypertrophic Cardiomyopathy via Protein Kinase D. Front. Physiol. 11, (2020).

28. Xu, M. et al. Altered Tyrosine Phosphorylation of Cardiac Proteins Prompts Contractile Dysfunction in Hypertrophic Cardiomyopathy. (2020).

29. Kim, D. H. Global Proteomics and Phosphoproteomics of Heart Failure Patients Elucidate Etiologyspecific Signaling. (University of Toronto, 2019).

30. W, H. The multiple roles of titin in muscle contraction and force production. Biophys. Rev. 10, 1187-1199 (2018).

31. WA, L. Sense and stretchability: the role of titin and titin-associated proteins in myocardial stress-sensing and mechanical dysfunction. Cardiovasc. Res. 77, 637-648 (2008).

32. KA, R. et al. Network reconstruction and systems analysis of cardiac myocyte hypertrophy signaling. $J$. Biol. Chem. 287, 42259-42268 (2012).

33. PM, T., KS, B., JH, O., AD, M. \& JJ, S. Predictive model identifies key network regulators of cardiomyocyte mechano-signaling. PLoS Comput. Biol. 13, (2017).

34. CA, T., ME, H., O, S., MRG, T. \& L, M. The Giant Protein Titin's Role in Cardiomyopathy: Genetic, Transcriptional, and Post-translational Modifications of TTN and Their Contribution to Cardiac Disease. Front. Physiol. 10, (2019).

35. F, S. et al. An FHL1-containing complex within the cardiomyocyte sarcomere mediates hypertrophic biomechanical stress responses in mice. J. Clin. Invest. 118, 3870-3880 (2008).

36. K, H. et al. A mutation in the human phospholamban gene, deleting arginine 14, results in lethal, hereditary cardiomyopathy. Proc. Natl. Acad. Sci. U. S. A. 103, 1388-1393 (2006).

37. MJ, K., AR, S. \& JJ, S. Modeling cardiac $\beta$-adrenergic signaling with normalized-Hill differential equations: comparison with a biochemical model. BMC Syst. Biol. 4, (2010).

38. GS, L. et al. A novel human R25C-phospholamban mutation is associated with super-inhibition of calcium cycling and ventricular arrhythmia. Cardiovasc. Res. 107, 164-174 (2015).

39. M, Y., Y, I. \& M, M. Altered intracellular Ca2+ handling in heart failure. J. Clin. Invest. 115, 556-564 (2005).

40. K, M. et al. C-type natriuretic peptide moderates titin-based cardiomyocyte stiffness. JCI insight 5, (2020).

41. BA, M. et al. Molecular and structural transition mechanisms in long-term volume overload. Eur. J. Heart Fail. 18, 362-371 (2016).

42. SF, N. et al. Altered titin expression, myocardial stiffness, and left ventricular function in patients with dilated cardiomyopathy. Circulation 110, 155-162 (2004).

43. Z, Z. Sensitivity analysis approaches applied to systems biology models. IET Syst. Biol. 5, 336-346 (2011).

44. CL, A. et al. Dilated cardiomyopathy and sudden death resulting from constitutive activation of protein kinase a. Circ. Res. 89, 997-1004 (2001).

45. Dimitrow, P. P., Undas, A., Bober, M., Tracz, W. \& Dubiel, J. S. Plasma biomarkers of endothelial dysfunction in patients with hypertrophic cardiomyopathy. Pharmacol. Reports 59, 715 (2007).

46. Y, L., WH, B., J, Z. \& F, S. Four and a half LIM domain protein signaling and cardiomyopathy. Biophys. Rev. 10, 1073-1085 (2018).

47. JC, B. et al. PKC-alpha regulates cardiac contractility and propensity toward heart failure. Nat. Med. 10, 248-254 (2004).

48. LA, G. et al. Hypotonic swelling promotes nitric oxide release in cardiac ventricular myocytes: impact on swelling-induced negative inotropic effect. Cardiovasc. Res. 104, 456-466 (2014).

49. Q, Z., PM, S., Y, H., J, T. \& HR, W. Cyclic GMP signaling and regulation of SERCA activity during cardiac myocyte contraction. Cell Calcium 37, 259-266 (2005).

50. J, I. \& D, G.-D. The cGMP/PKG pathway as a common mediator of cardioprotection: translatability and 
mechanism. Br. J. Pharmacol. 172, 1996-2009 (2015).

51. S, F. et al. Stress-dependent dilated cardiomyopathy in mice with cardiomyocyte-restricted inactivation of cyclic GMP-dependent protein kinase I. Eur. Heart J. 34, 1233-1244 (2013).

52. S, M. et al. Chronic phospholamban-sarcoplasmic reticulum calcium ATPase interaction is the critical calcium cycling defect in dilated cardiomyopathy. Cell 99, 313-322 (1999).

53. Rationalizing combination therapies. Nat. Med. 23, 1113 (2017).

54. E, K. Sacubitril/valsartan in heart failure: latest evidence and place in therapy. Ther. Adv. Chronic Dis. 7, 278-290 (2016).

55. PS, D. et al. RAF1 mutations in childhood-onset dilated cardiomyopathy. Nat. Genet. 46, 635-639 (2014).

56. CY, H. et al. Diltiazem treatment for pre-clinical hypertrophic cardiomyopathy sarcomere mutation carriers: a pilot randomized trial to modify disease expression. JACC. Heart Fail. 3, 180-188 (2015).

57. C, S. et al. The L-type calcium channel inhibitor diltiazem prevents cardiomyopathy in a mouse model. $J$. Clin. Invest. 109, 1013-1020 (2002).

58. CW, Y. et al. 2017 ACC/AHA/HFSA Focused Update of the 2013 ACCF/AHA Guideline for the Management of Heart Failure: A Report of the American College of Cardiology/American Heart Association Task Force on Clinical Practice Guidelines and the Heart Failure Society of America. Circulation 136, e137-e161 (2017).

59. JR, R. F., JN, C., CM, C. \& AC, P.-B. Reverse Cardiac Remodeling: A Marker of Better Prognosis in Heart Failure. Arq. Bras. Cardiol. 104, 502-506 (2015).

60. T, Y. et al. A new therapeutic strategy for hypertrophic nonobstructive cardiomyopathy in humans. A randomized and prospective study with an Angiotensin II receptor blocker. Int. Heart J. 48, 715-724 (2007).

61. M, P. et al. The effects of candesartan on left ventricular hypertrophy and function in nonobstructive hypertrophic cardiomyopathy: a pilot, randomized study. J. Mol. Diagn. 11, 35-41 (2009).

62. H, K. et al. Valsartan decreases type I collagen synthesis in patients with hypertrophic cardiomyopathy. Circ. J. 69, 1244-1248 (2005).

63. S, S. et al. Mavacamten Favorably Impacts Cardiac Structure in Obstructive Hypertrophic Cardiomyopathy: EXPLORER-HCM Cardiac Magnetic Resonance Substudy Analysis. Circulation 143, 606-608 (2021).

64. W, G. \& M, S. RBM20, a potential target for treatment of cardiomyopathy via titin isoform switching. Biophys. Rev. 10, 15-25 (2018).

65. C, H. et al. PKC phosphorylation of titin's PEVK element: a novel and conserved pathway for modulating myocardial stiffness. Circ. Res. 105, 631-638 (2009).

66. NW, L. \& PJ, C. Principles of multiparametric optimization for phospholipidomics by 31 P NMR spectroscopy. Biophys. Rev. 5, 295-304 (2013).

67. JR, E. Mechanisms of CaMKII Activation in the Heart. Front. Pharmacol. 5, (2014).

68. S, L., W, G., CN, D. \& ML, G. Rbm20 regulates titin alternative splicing as a splicing repressor. Nucleic Acids Res. 41, 2659-2672 (2013).

69. M, M. et al. Experimentally increasing titin compliance in a novel mouse model attenuates the FrankStarling mechanism but has a beneficial effect on diastole. Circulation 129, 1924-1936 (2014).

70. M, I. et al. The Akt-mTOR axis is a pivotal regulator of eccentric hypertrophy during volume overload. Sci. Rep. 5, (2015).

71. T, Y. et al. Clinical impact of myocardial mTORC1 activation in nonischemic dilated cardiomyopathy. $J$. Mol. Cell. Cardiol. 91, 6-9 (2016).

72. I, M. et al. Passive stiffness changes caused by upregulation of compliant titin isoforms in human dilated cardiomyopathy hearts. Circ. Res. 95, 708-716 (2004).

73. K, N. et al. Calcium-dependent titin-thin filament interactions in muscle: observations and theory. $J$. Muscle Res. Cell Motil. 41, 125-139 (2020).

74. D, L. et al. Calcium-dependent molecular spring elements in the giant protein titin. Proc. Natl. Acad. Sci. U.S. A. 100, 13716-13721 (2003).

75. TR, L. \& W, H. Regulation of muscle force in the absence of actin-myosin-based cross-bridge interaction. 
Am. J. Physiol. Cell Physiol. 299, (2010).

76. WA, L. \& M, K. The giant protein titin as an integrator of myocyte signaling pathways. Physiology (Bethesda). 25, 186-198 (2010).

77. FW, F. et al. FHL2 expression and variants in hypertrophic cardiomyopathy. Basic Res. Cardiol. 109, (2014).

78. B, H., BA, R., TG, G. \& JA, H. FHL2 binds calcineurin and represses pathological cardiac growth. Mol. Cell. Biol. 32, 4025-4034 (2012).

79. F, K.-S. et al. Cardiac thin filament regulation and the Frank-Starling mechanism. J. Physiol. Sci. 64, 221232 (2014).

80. JK, F. \& WA, L. Titin as a force-generating muscle protein under regulatory control. J. Appl. Physiol. 126, 1474-1482 (2019).

81. BD, W., AG, E., AD, M. \& JH, B. The promise of CaMKII inhibition for heart disease: preventing heart failure and arrhythmias. Expert Opin. Ther. Targets 17, 889-903 (2013).

82. MY, M., HA, K. \& J, B. Regulation of CaMKII signaling in cardiovascular disease. Front. Pharmacol. 6, (2015).

83. D, L., J, B. \& MMG, van den H. New Insights in RBM20 Cardiomyopathy. Curr. Heart Fail. Rep. 17, 234-246 (2020).

84. F, C. \& K, L. Inhibition of cardiac CaMKII to cure heart failure: step by step towards translation? Basic Res. Cardiol. 111, (2016).

85. AJ, B. et al. Calcium/Calmodulin-Dependent Protein Kinase II Delta Inhibition and Ventricular Remodeling After Myocardial Infarction: A Randomized Clinical Trial. JAMA Cardiol. (2021) doi:10.1001/JAMACARDIO.2021.0676.

86. SJ, L. et al. Chronic Calmodulin-Kinase II Activation Drives Disease Progression in Mutation-Specific Hypertrophic Cardiomyopathy. Circulation 139, 1517-1529 (2019).

87. TH, F. et al. $\mathrm{Ca}(2+) /$ calmodulin-dependent protein kinase II equally induces sarcoplasmic reticulum $\mathrm{Ca}(2+)$ leak in human ischaemic and dilated cardiomyopathy. Eur. J. Heart Fail. 16, 1292-1300 (2014).

88. X, F. et al. Celecoxib and 2,5-dimethyl-celecoxib prevent cardiac remodeling inhibiting Akt-mediated signal transduction in an inherited dilated cardiomyopathy mouse model. J. Pharmacol. Exp. Ther. 338, 2$11(2011)$.

89. KL, W., BC, B., JYY, O., NL, P. \& JR, M. The IGF1-PI3K-Akt Signaling Pathway in Mediating ExerciseInduced Cardiac Hypertrophy and Protection. Adv. Exp. Med. Biol. 1000, 187-210 (2017).

90. L, G. et al. Isorhamnetin protects against cardiac hypertrophy through blocking PI3K-AKT pathway. Mol. Cell. Biochem. 429, 167-177 (2017).

91. K, P. et al. Novel EGFR inhibitors attenuate cardiac hypertrophy induced by angiotensin II. J. Cell. Mol. Med. 20, 482-494 (2016).

92. W, A. et al. Silibinin attenuates cardiac hypertrophy and fibrosis through blocking EGFR-dependent signaling. J. Cell. Biochem. 110, 1111-1122 (2010).

93. T, A. \& T, M. Phosphoinositide-3 kinase signaling in cardiac hypertrophy and heart failure. Curr. Pharm. Des. 17, 1818-1824 (2011).

94. T, S. et al. Rapamycin attenuates load-induced cardiac hypertrophy in mice. Circulation 107, 1664-1670 (2003).

95. TM, M. et al. Rapamycin reverses hypertrophic cardiomyopathy in a mouse model of LEOPARD syndrome-associated PTPN11 mutation. J. Clin. Invest. 121, 1026-1043 (2011).

96. M, M. et al. Experimentally Increasing the Compliance of Titin Through RNA Binding Motif-20 (RBM20) Inhibition Improves Diastolic Function In a Mouse Model of Heart Failure With Preserved Ejection Fraction. Circulation 134, 1085-1099 (2016).

97. KR, H., C, S., CS, C. \& H, G. Increased myocardial stiffness due to cardiac titin isoform switching in a mouse model of volume overload limits eccentric remodeling. J. Mol. Cell. Cardiol. 79, 104-114 (2015).

98. C, S. et al. Metabolic Alterations in Inherited Cardiomyopathies. J. Clin. Med. 8, 2195 (2019).

99. J, Z. et al. Titin truncations lead to impaired cardiomyocyte autophagy and mitochondrial function in vivo. Hum. Mol. Genet. 28, 197-1981 (2019). 
100. JAJ, V. et al. Titin cardiomyopathy leads to altered mitochondrial energetics, increased fibrosis and longterm life-threatening arrhythmias. Eur. Heart J. 39, 864-873 (2018).

101. Z, G. et al. Amelioration of desmin network defects by $\alpha \mathrm{B}$-crystallin overexpression confers cardioprotection in a mouse model of dilated cardiomyopathy caused by LMNA gene mutation. J. Mol. Cell. Cardiol. 125, 73-86 (2018).

102. H, A., C, R., E, B. \& H, W. Hypertrophic cardiomyopathy:a paradigm for myocardial energy depletion. Trends Genet. 19, 263-268 (2003).

103. NG, F. The Extracellular Matrix in Ischemic and Nonischemic Heart Failure. Circ. Res. 125, 117-146 (2019).

104. Khare, Y. P., Muñoz-Carpena, R., Rooney, R. W. \& Martinez, C. J. A multi-criteria trajectory-based parameter sampling strategy for the screening method of elementary effects. Environ. Model. Softw. 64, 230-239 (2015). 